\title{
Les signaux faibles : du mythe à la mystification
}

\author{
Camille Alloing \& Nicolas Moinet
}

\section{Cet article est une version « pre-print », certains éléments peuvent ainsi différer de celui publié.}

Merci de citer : ALLOING, C., Moinet, M., « Les signaux faibles : du mythe à la mystification », Hermès $n^{\circ} 76,2016$, pp. 68-76

Quiconque aura réalisé une revue de l'actualité sur la question du renseignement dans les attentats terroristes qui ont particulièrement touché la France depuis janvier 2015 et mené une analyse des discours, retiendra que la clé de l'anticipation réside dans le repérage des «signaux faibles », ces micro-évènements annonciateurs d'une atteinte grave à l'intégrité d'une personne ou d'un groupe, d'une organisation ou d'un territoire. Par leur collecte et leur traitement approprié, ceux-ci permettraient de prévenir des menaces imminentes et de contrecarrer les plans des réseaux djihadistes autant que d'empêcher le passage à l'acte des fameux «loups solitaires ». Comment ne pas abonder dans ce sens ? Plus la vigilance sera forte et les signaux faibles amplifiés et plus la ré-action pourra se transformer en pro-action, l'étape suivante étant celle de la pré-diction symbolisée par le film Minority Report ${ }^{l}$. En conclusion: Vive les signaux faibles ! Et tous à nos écrans radar!

Oui mais voilà. Quiconque aura réalisé une revue de l'actualité sur la question du renseignement dans les attentats terroristes et dépassé les discours pour leur préférer la froide analyse des faits et de leur enchaînement, en sera arrivé à la conclusion que les signaux faibles n'ont pas été repérés par les services spécialisés, soit parce que ces derniers n'en n'ont pas (encore) les capacités, soit parce que ces dits signaux sont par essence quasiment impossibles à repérer (puisque faibles), soit encore par qu'ils n'existent tout simplement pas ou, dit de manière plus scientifique, parce qu'ils ne constituent pas un concept opératoire. C'est cette dernière thèse que nous défendrons ici en montrant comment le signal faible est passé du mythe à la mystification et combien - loin de n'être qu'une querelle sémantique son usage dans certains contextes peut s'avérer contre-productif, irresponsable et même dangereux. Pour cela, nous partirons de la genèse de cette notion, expliquerons les limites de sa transposition et analyserons les forces qui ont abouti à sa mutation. Cette radioscopie nous permettra, au final, de proposer une autre approche où la pertinence conceptuelle induit la performance opérationnelle, faisant nôtre cette idée de Kurt Lewin selon laquelle «Rien n'est plus pratique qu'une bonne théorie ».

\footnotetext{
${ }^{1}$ Ce film de Steven Spielberg (2002) nous conduit en 2054 dans une ville de Washington qui a réussi à éradiquer la criminalité. Grâce aux visions du futur fournies par trois individus exceptionnels doués de précognition, les agents de Précrime peuvent arrêter les criminels juste avant qu'ils ne commettent leurs méfaits.
} 


\section{Analyse d'une mystification}

Dès lors que l'on suit l'actualité du renseignement et que l'on analyse plus scientifiquement les méthodes mises en œuvre, la pertinence des signaux faibles tend à s'affaiblir jusqu'à disparaître. Après une analyse de l'actualité et des discours, nous présentons en guise d'illustration trois articles du premier quotidien national qui - par leur proximité temporelle (après l'attentat de Nice du 14 juillet 2016) - permettent de mieux comprendre le fonctionnement mystificateur du concept de signal faible.

«Terrorisme : le gouvernement n'a-t-il vraiment rien fait depuis deux ans? Pour faciliter les poursuites contre les «loups solitaires», qui se radicalisent en dehors d'un réseau ou d'une organisation terroriste, le délit d'entreprise terroriste individuelle a été créé. Il peut être caractérisé par plusieurs "signaux faibles » avant le passage à l'acte, comme la détention d'armes, les séjours dans des zones de djihad ou la consultation répétée de sites faisant l'apologie du terrorisme. » (Untersinger, 2016). Ici, on remarquera que sont qualifiés de signaux faibles des actes majeurs et que la faiblesse du signal est toute relative ! Mais surtout, comment expliquer qu'un 19 tonnes (qui fera donc plus de 80 morts et 200 blessés en 45 secondes sur la promenade des anglais le soir du feu d'artifice de la Fête Nationale) ait été contrôlé et non arrêté quand on sait notamment que les camions sont interdits dans cette zone et que le chauffeur indiquait venir livrer des glaces alors que son camion n'était pas frigorifique ? Le couple cohérence/incohérence est pourtant la base de toutes les démarches d'investigation. En fait, l'usage dans les discours de la notion de signal faible est à rapprocher ici de celle de «loup solitaire». Mais «Les loups du djihad sont-ils si solitaires?» questionne justement un spécialiste du terrorisme. Et de répondre : «Bien souvent, il a même été établi qu'une poignée de personnes autour du terroriste étaient informées du projet, ou étaient assez proches de lui pour qu'on puisse envisager l'existence d'une petite base arrière. La question, désormais centrale, est de savoir comment détecter puis neutraliser à temps de tels individus, à partir de quels signaux faibles et selon quelle procédure légale. La figure des loups solitaires est le plus souvent invoquée abusivement. On a ainsi beaucoup dit que Mohamed Merah, le tueur de Toulouse et de Montauban, en 2012, en était un. Rien n'est pourtant moins vrai, et il a été largement démontré que Merah, dont les crimes ont d'ailleurs été revendiqués, agissait sur ordre, certes de façon autonome, mais pas sans lien. La confusion avec des tactiques mises en avant depuis près de quinze ans par les penseurs du djihad a ici été utilisée pour masquer un échec. » Et ce qui est vrai de l'usage abusif de la notion de «loups solitaires » l'est également de celle de « signal faible».

Sinon, comment expliquer que des signaux dits faibles, dont on sait a posteriori qu'ils avaient été recensés, ne permettent pas aux services de renseignement d'anticiper de futurs attentats ? D'autant qu'une analyse des trajectoires de la plupart des terroristes montre que ceux-ci étaient la plupart du temps connus des services, généralement «fichés $S$ » et même parfois considérés comme cibles prioritaires. Une question de compétence? Parfois sans doute comme dans toutes les activités, mais le professionnalisme des services de renseignement est élevé. Une histoire de moyens ? Sans ignorer le manque de moyens de certains services - à commencer par le renseignement dit territorial - la principale clé se trouve néanmoins ailleurs selon nous... dans le caractère non opératoire du concept de signal faible. 
"Les terroristes frapperont là où il y a une symbolique qui n'a pas encore été prise en compte », croit pour sa part une source au sein des services de renseignement, pour qui une des difficultés réside dans l'appréhension des signaux faibles. En conséquence, "le tamis du filet se resserre beaucoup ", constate Patrice Ribeiro, secrétaire général du syndicat de policiers Synergie officiers. "Certains signalements n'auraient pas été pris en compte aussi sérieusement il y a un an. Ce n'est pas de l'hystérie, c'est paradigmatique. La menace est telle que les services sont sur un principe de précaution ». (Belouezzane, 2016). Ici, le signal faible fonctionne comme un bouclier permettant de justifier tous les contrôles, le principe de précaution posant évidemment question à l'Etat de droit. Mais pourquoi pas s'il est efficace, diront les tenants d'une ligne pragmatique ? Le fait est que les récents attentats, déjoués ou non, ne l'ont pas été en raison d'une captation de signaux faibles. Ainsi, ce n'est pas la soudaine radicalisation de l'une des meneuses (fichée S) de la tentative d'attentat à NotreDame de Paris qui a alerté les services mais bien la voiture stationnée depuis deux jours à côté de la Cathédrale et dans laquelle se trouvaient cinq bombonnes de gaz qui n'explosèrent pas faute de compétence des terroristes. Mais il y a pire : loin de ne pas être repérés, les signaux faibles viennent même désorganiser le renseignement, aux dires de certains de ses responsables.

«Depuis le début de l'année 2016, la police lyonnaise aurait traité près de quarante procédures pour apologie du terrorisme. Le chef de la sûreté départementale du Rhône note que les infractions quotidiennes à connotations terroristes sont en augmentation, mais qu'il convient d'analyser le phénomène avec prudence car l'extrême attention des autorités produirait aussi un effet de loupe: "Nous traitons tous les événements sans rien laisser passer, le travail policier doit désormais ratisser tous les signaux, ce qui peut donner l'impression de surréagir sur des faits qui, avant les attentats, auraient pu paraître banals au quotidien. » (Schittly, 2016). Inefficaces, les signaux faibles pourraient même s'avérer contreproductifs. Ils seraient alors comme le visage de la personne attendue dans un lieu public : plus l'attente se prolonge et plus les visages des gens qui passent lui ressemblent. Mais alors, comment expliquer la diffusion d'un concept non opératoire ? L'a-t-il toujours été et si non, dans quel contexte?

\section{Petite histoire d'un mythe}

Importée des Etats-Unis et plus spécifiquement de la littérature sur la stratégie et le management des entreprises, cette notion de signal faible est notamment bien connue des spécialistes de l'intelligence économique qui partagent avec les agents des services de renseignement une part commune de culture et de méthodologie.

En 1975, Igor Ansoff présente les signaux faibles, pour les organisations commerciales, comme des éléments permettant d'anticiper toute «surprise stratégique ». Les détecter offrirait aux décideurs la possibilité d'anticiper les conséquences d'événements difficilement prévisibles, et surtout menaçants la survie économique de l'organisation. Dès les prémices de cette notion, nous pouvons ainsi remarquer que celle-ci est promue par des arguments anxiogènes : les signaux faibles existent, puisque de manière rétroactive il est possible de les identifier, et ils représentent un risque, d'où la nécessité de les capter. En 1984, Ansoff et MacDonnell définissent le signal faible comme «Un fait à propos duquel seules des informations partielles sont disponibles alors qu'une réaction doit être entamée, si l'on veut 
qu'elle soit parachevée avant impact sur la firme de l'évènement nouveau» (cité par Castagnos, et Lesca, 2004). Le fait que les informations soient «partielles » fait dire à Humbert Lesca (2003) que nous sommes plus proches de l'intuition pour caractériser une information ou un signal dit « faible ».

La question serait alors moins de pouvoir le détecter, que de décider que telle bribe d'information nécessite ou non d'être étudiée de manière précise. Ce qui fait dire à Blanco (1998, cité par Lesca, 2000) que plus que des signaux (perceptible de manière homogène par chaque récepteur et reposant sur une intentionnalité de la part de l'émetteur), il convient de traiter de «signes », dont le sens reste négociable. L'interprétation possible de ces signes, le contexte dans lequel ils sont émis et reçus, la situation d'urgence ou d'incertitude dans laquelle se trouve le décideur qui doit s'appuyer sur eux, sont des éléments centraux qui amènent à souligner qu'il y aurait autant de « signes faibles » que d'émetteurs communicants une même information et de récepteurs venant la traiter. Le signal faible est «paradoxal» (Cahen, 2011). Il répond à une intentionnalité, un regard et une attention qui ne peuvent être que subjectifs. Qui plus est lorsque le postulat de départ est qu'un événement surviendra quoi qu'il arrive, et qu'il est nécessaire, voire vital, de le prévenir. Il apparaît alors que le signal faible est un concept opératoire dans le champ de la prospective. Mais est-il tout aussi opératoire dans le champ de la surveillance (veille, renseignement) ? Le paradoxal mis en exergue est-il pertinent dans d'autres cadres que celui d'une anticipation des ruptures ? Le fait est que dans la communauté professionnelle du renseignement (au sens large, c'est-à-dire incluant les professionnels de la veille et de l'intelligence économique), les signaux faibles vont devenir un mythe, c'est-à-dire un ensemble de croyances et de représentations idéalisées autour d'un objet, puis d'outils de captation, et partagées par un groupe qui renforce son identité à travers lui. Mythe ne doit pas être connoté négativement, du moins dans la phase de consolidation d'une communauté. Mais il peut devenir contre-productif avec le temps dès lors qu'il fige les pratiques et empêche toute remise en question. Tel est bien le cas des signaux faibles qui racontent une histoire, celle d'une organisation capable d'anticiper les évolutions de son environnement en captant - après les avoir éventuellement amplifié - des signaux faibles. Voir avant tout le monde ce que les autres ne voient pas encore en rationalisant ce qui ne peut être pourtant rationalisé dans le processus de décision - l'intuition et la subjectivité et en limitant autant que possible le risque pourtant consubstantiel à tout choix. On le pressent, plusieurs acteurs vont se retrouver dans les signaux faibles : les veilleurs qui doivent légitimer leurs fonctions, les manageurs qui redoutent les incertitudes de l'avenir, les services de sécurité qui rêvent de réduire le risque ou encore les consultants et fournisseurs de solutions logicielles qui voient s'ouvrir un marché pour leurs méthodes et outils. Des signaux faibles vous ont échappé ? Rassurez-vous. Nous avons désormais le radar qui vous convient...

Tout d'abord, remarquons que si ces signaux faibles sont si importants, pourquoi ne pas déjà traiter les signaux forts ? Accroissement des catastrophes naturelles, fin annoncée du pétrole, surendettement structurel des Etats, désindustrialisation chronique, désertification médicale, «burn-out» des enseignants, baisse du niveau scolaire, fuite des cerveaux, perte de compétitivité, vieillissement de la population, risques nucléaires, etc. Quels que soient les domaines, comment expliquer que les décideurs ne traitent pas plus rapidement ces signaux dits forts, attendant le plus souvent la crise ou l'accident pour ré-agir ? Le syndrome du Titanic a décidément de beaux jours devant lui. Pourtant averti de la présence d'icebergs, 
l'équipage du transatlantique n'avait pas su les éviter. La suite est connue... et la réponse est à chercher du côté du processus de décision et de la notion de connaissance comme produit individuel et collectif issus d'informations et guidé par une vision commune du but à atteindre et du sens à donner à l'action (Moinet, 2011).

\section{Les signaux faibles comme convention : l'arrivée du « big data » et des algorithmes}

Le recours aux dispositifs numériques, et spécifiquement pour le traitement des grands jeux de données auxquels ils donnent accès («big data ») est aujourd'hui central dans la chaine de traitement amenant à produire de l'information. Les « informations sur l'information » que sont les métadonnées, sont un enjeu pour les services de renseignement (appels téléphoniques, connexions à des plates-formes web, géolocalisation, etc.) comme pour les organisations (relations «d'amitiés » sur les réseaux socionumériques, temps passé à regarder une vidéo, etc.). Ces deux entités, services et organisations, s'équipent de nombreux outils logiciels afin de capter et traiter des forts volumes de données produits de manière ordinaire par chaque usager de dispositifs connectés. Ainsi, de nombreux éditeurs de logiciels de veille proposent de détecter des signaux faibles (Alloing, 2016) par comparaison des volumes d'information diffusés en lignes (ce qui n'est pas fort est faible), mais aussi par le profilage comportemental des usagers de certaines plates-formes : après avoir défini un profil type de consommateur, sera jugé comme un signal faible tout comportement "déviant» en provenance d'un consommateur jugé comme «influent» ou «représentatif» d'un groupe donné. La «politique des grands nombres» et la «raison statistique » d'Alain Desrosières (2016) s'appuient sur un allié de taille (le web) et sur des technologies de calcul dont les capacités ne cessent de s'accroitre. Il serait bien trop long de lister l'ensemble des promesses et applications du «big data » d'un point de vue commercial. Un élément qui semble récurrent, et qui n'a pas échappé aux services de renseignement tant il est lié à la notion de signal faible, est celui de la prédiction. Le logiciel «Predpol» (utilisé notamment par la police de d'Atlanta) propose, par exemple, d'aider les policiers à anticiper les crimes à venir en compilant et en traitant les statistiques de criminalité existantes (www.predpol.com).

Ce traitement repose sur des algorithmes fonctionnant en « boite noire » et s'appuyant sur des modèles mathématiques variés. Lors des débats sur la «loi renseignement» en France en 2015, le gouvernement a ainsi mis en avant sa volonté d'installer «chez les fournisseurs d'accès et certains grands sites Internet, [un] dispositif d'analyse automatique de données créé par les services de renseignement et censé, selon la formule du projet de loi, "révéler une menace terroriste» de manière automatisée» (Le Monde, 2015). Comme pour les signaux faibles, il convient de souligner que ce traitement automatisé de grands jeux de données suppose de manipuler des signaux et non des signes. Le contexte n'est plus simplement mis de côté mais homogénéisé afin de favoriser le calcul des données collectées. De plus, et comme le mettent en exergue Rouvroy et Berns (2013), le calcul algorithmique désubjectivise les sujets afin de les rendre calculables. En somme, les algorithmes de traitement des données ne pouvant analyser des signes, ils produisent des signaux. Cependant, si dans un objectif commercial il peut s'avérer intéressant de créer un contexte favorable à partir de prédictions en provenance de données désubjectivisées et de signaux fabriqués par un traitement statistique, autrement dit de transformer la faiblesse du signal en tendance, l'on 
peut s'interroger sur la pertinence de telles approches dans un contexte de renseignement. Comme le souligne Bilel Benbouzid (2016) à propos de «Predpol»: «Les techniques de marketing utilisées réduisent les discussions à de la persuasion. ». Le mythe du signal faible semble alors être soluble dans les nouvelles conventions concernant les algorithmes prédictifs. Ces conventions associent à la fois le caractère auto-réalisateur de certaines approches en prospective, les pratiques parfois bien ancrées de gouvernance par la statistique, et les discours souvent prophétiques des acteurs dominants du Web. La question du sens, pourtant essentielle à la prise de décision, céderait-elle le pas à celle de la performance immédiate ?

\section{Conclusion : ce n'est pas le signal qui est faible, c'est l'intelligence}

Dès les fondations de l'intelligence économique, tant chez les auteurs anglo-saxons que pour les initiateurs du rapport Martre, il va de soi que l'information seule ne permet pas d'agir. Chez Wilensky (1967), l'intelligence est entendue comme le recueil, l'interprétation et la valorisation systématique de l'information pour la poursuite de ses buts stratégiques. Pour l'auteur américain, il ne s'agit pas d'un processus d'accumulation d'informations mais plutôt de production de connaissances, par les gouvernements et les industriels, et quand nécessaire, dans le cadre de stratégies collectives. Et le véritable levier concurrentiel et stratégique se trouve dans les compétences d'interprétation (interpretation skills) quand les principaux obstacles sont les rigidités organisationnelles (hiérarchisation et bureaucratisation en particulier). Mais une fois passée cette mise en garde, le quotidien reprend le dessus et c'est le concept d'information qui domine. Pourtant, les conceptions mécanistes de l'information citées précédemment vont marquer durablement les pratiques de leur empreinte. Ainsi, les années 90 vont voir naître dans les organisations de nombreuses cellules de veille à la recherche de l'information dite stratégique et de nos fameux signaux faibles. Pourtant, le rapport fondateur de l'intelligence économique, travail collectif produit par un groupe d'experts précisait: «Beaucoup d'entreprises ayant créé un département centralisé d'intelligence économique ont échoué dans leur démarche. Il s'avère que les capacités de comprendre et de savoir liées à l'intelligence économique se diffusent de façon informelle et s'apparentent à un processus. L'action d'une structure centralisée ne produit pas l'intensité de connaissances suffisante pour l'appréhension effective d'environnements complexes, ni ne permet sa diffusion rapide ». (Martre, 1994, p 69).

Si la notion de signal faible a pu, dans un premier temps, permettre la mise en tension des organisations, elle est désormais contre-productive dans de nombreux domaines car conduit à des modes d'organisation qui sont contraires à son esprit. Dans une vision prospective visant à anticiper les mutations, le signal faible devait permettre à une organisation de réfléchir collectivement aux éléments annonciateurs de ruptures. Mais sous l'influence d'organisations pyramidales visant un contrôle en réalité inatteignable, et le développement de conventions quant à la pertinence du traitement automatisé de grands jeux de données, le nécessaire travail de mise en commun d'interprétations variées d'un même événement semble s'effacer.

Le sujet des signaux faibles est donc l'arbre qui cache la forêt. Au-delà se pose finalement la question du rapport entre un monde du renseignement, fermé par nécessité et une communauté académique, ouverte par essence. 


\section{Bibliographie}

AlloING, C., (E)réputation : médiation, calcul, émotion, CNRS Edition, 05/2016

ANSOFF, H.I., « Managing strategic surprise by response to weak signals », California Management Review, 1975. Vol. 18 n² pp. 21-33

Ansoff, H. I., Mc Donnell, E., « Implanting Strategic Management », Prentice Hall International, Englewood Cliffs, NY, 1990 (1ère edition 1984)

Belouezzane, S., Faye, O. Seelow S., Pascual J. et Deffrennes, G., «Terrorisme : l'inquiétude s'installe à son plus haut niveau », Le Monde, 6 août 2016

BenbouZID, B., «À qui profite le crime ? Le marché de la prédiction du crime aux ÉtatsUnis », La vie des idées, 13/09/16. En ligne : http://www.laviedesidees.fr/A-qui-profite-lecrime.html

BlanCO, S., Gestion de l'information et intelligence stratégique : cas de la sélection des signes d'alerte précoce de veille stratégique. Thèse de doctorat en Science de gestion, Grenoble 2 UPMF, ESA, CERAG, 1998, 307 p.

CAHEN, P., Signaux faibles : mode d'emploi, Eyrolles, 2011

Castagnos, J-C. et LesCA, H., Capter les signaux faibles de la veille stratégique : retours d'expérience et recommandations. E \& G, Economia et Gestäo, Belo Horizonte, v.4, n.7, pp. 15-34, 2004

DESROSIÈRES, A., La politique des grands nombres: histoire de la raison statistique, Editions La découverte, 2016

LESCA, H., Veille stratégique. La méthode L.E. Scanning, Editions EMS, 2003

LeSCA, N., «Processus de construction du sens à partir de signes d'alerte précoce: proposition d'un nouvel outil d'aide à la production de connaissance : PUZZLE », Actes de

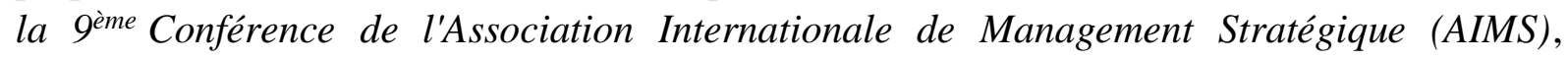
Montpellier, 24-26 mai, 2000, 22 p.

MARTRE, H. (Dir.), Intelligence économique et stratégie des entreprises, Rapport du groupe de travail du Commissariat Général du Plan, La Documentation Française, 1994

MoINET, N., Intelligence économique. Mythes \& réalités, CNRS Editions, 2011

Rouvroy, A. et BERNS, T., «Gouvernementalité algorithmique et perspectives d'émancipation », Réseaux, 2013, no 1, pp. 163-196

Schittly, R., «Dans le contexte terroriste, plus d'incidents et de fausses alertes. », Le Monde, 9 août 2016

UNTERSINGER, M., «Loi sur le renseignement : la «boîte noire » reste obscure », Le Monde, $1^{\text {er }}$ avril 2015

Untersinger, M., VAudano, M., Boscher, M., «Terrorisme : le gouvernement n'a-t-il vraiment rien fait depuis deux ans ? », Le Monde, 18 juillet 2016

Wilensky, H., Organizational Intelligence: Knowledge and Policy in Government and Industry, New York, Basic Books, 1967 